\title{
Influence of Different Cultural Conditions on Photoproduction of Hydrogen by Rhodopseudomonas palustris KU003
}

\author{
Ramchander Merugu, ${ }^{1}$ M. P. Pratap Rudra, ${ }^{2}$ A. Sridhar Rao, ${ }^{1}$ D. Ramesh, ${ }^{1}$ \\ B. Nageshwari, ${ }^{1}$ K. Rajyalaxmi, ${ }^{3}$ S. Girisham, ${ }^{3}$ and S. M. Reddy ${ }^{3}$ \\ ${ }^{1}$ University College of Science and Informatics, Mahatma Gandhi University, Nalgonda 508001, India \\ ${ }^{2}$ Department of Biochemistry, Osmania Unviersity, Hyderabad 500007, India \\ ${ }^{3}$ Department of Biochemistry and Microbiology, Kakatiya University, Warangal 506009, India
}

Correspondence should be addressed to Ramchander Merugu, rajumerugu01@rediffmail.com

Received 8 October 2011; Accepted 23 November 2011

Academic Editor: A. Bosio

Copyright (C) 2011 Ramchander Merugu et al. This is an open access article distributed under the Creative Commons Attribution License, which permits unrestricted use, distribution, and reproduction in any medium, provided the original work is properly cited.

Photoproduction of hydrogen by Rhodopseudomonas palustris KU003 under different cultural conditions with various carbon and nitrogen sources was investigated. Hydrogen production was measured using a Gas chromatograph. Malate promoted more amounts of hydrogen production under anaerobic light conditions than anaerobic dark conditions. Cumulative hydrogen production by the organism was recorded at various time intervals. Incubation period of $120 \mathrm{hrs}$ was optimum for production of hydrogen. $\mathrm{pH} 7.0 \pm 0.4$ was optimum for production of hydrogen. L-glutamic acid was a good nitrogen source for production of hydrogen. Growing cells produced more amount of hydrogen than resting cells. Significance of the above results in presence of existing literature is discussed.

\section{Introduction}

Hydrogen is considered as a potential fuel for the future because it is renewable, ecofriendly, cheaper, transportable, has high energy content for per unit mass of any known fuel, it is easily convertible to electricity by fuel cells, and on combustion it gives water as the only byproduct. Compared to nonphototrophic bacteria, phototrophic bacteria produce threefold greater amounts of hydrogen per mole of substrate utilized. When the conversion efficiency of substrate into hydrogen by chemotrophs restricted to $33.3 \%$, phototrophs can reach upto $100 \%$.

A wide variety of organic substrates such as carbohydrates $[1,2]$, lactate, malate, benzoate [3], sucrose [4], and nitrogen sources Ethanolamine [5] and L-cysteine [6] were reported to promote higher amounts of hydrogen in phototrophic bacteria. However, the substrate specificity for hydrogen production varied with the species [7]. Stimulation of hydrogen production by spraying nitrogen gas in the presence of glucose has been reported by Mizuno et al. [8]. The efficacy of different organic substrates for hydrogen production in R. rubrum was investigated by Melnicki et al. [7]. In view of the above facts, influence of different cultural conditions on hydrogen production by anoxygenic phototrophic bacteria was studied and the results are discussed.

\section{Material and Methods}

The phototrophic bacteria were isolated from the effluent samples by enrichment techniques by inoculating into the medium and incubated anaerobically in visible light $(2,000$ lux). Bacteria thus isolated were identified with the help of cultural characteristics (colour, size, and shape), carbon and nitrogen requirement, vitamin requirements, absorption spectra analysis, bacteriochlorophylls, and carotenoids. Identification keys provided in Bergey's manual of systematic bacteriology (1994) [9] was adopted. Growth was determined by measuring optical density at $660 \mathrm{~nm}$ using UV-Vis spectrophotometer.

2.1. Preparation of Growing Cells. Logarithmic cultures of $R$. palustris were inoculated $(1 \% \mathrm{v} / \mathrm{v})$ into basal medium containing different carbon sources (1\%) along with ammonium chloride $(0.5 \%)$ as nitrogen source. When different nitrogen 
TABLE 1: Effect of $\mathrm{pH}$ on hydrogen production ( $\mathrm{mL} / 15 \mathrm{~mL}$ vessel) by $R$. palustris.

\begin{tabular}{|c|c|c|c|c|c|c|c|c|c|}
\hline \multirow{2}{*}{$\mathrm{pH}$} & & \multicolumn{6}{|c|}{ Incubation period (in hrs) } & \multirow[b]{2}{*}{166} & \multirow[b]{2}{*}{192} \\
\hline & & 24 & 48 & 72 & 96 & 120 & 144 & & \\
\hline \multirow{2}{*}{5.0} & $\mathrm{R}$ & - & - & - & - & $0.22 \pm 0.06$ & - & - & - \\
\hline & G & - & - & $0.32 \pm 0.14$ & $0.50 \pm 0.12$ & $0.68 \pm 0.04$ & $0.44 \pm 0.08$ & - & - \\
\hline \multirow{2}{*}{5.5} & $\mathrm{R}$ & - & - & - & - & $0.25 \pm 0.04$ & $0.38 \pm 0.08$ & - & - \\
\hline & G & - & $0.24 \pm 0.04$ & $0.56 \pm 0.24$ & $0.94 \pm 0.16$ & $1.10 \pm 0.18$ & $0.78 \pm 0.08$ & $0.38 \pm 0.04$ & 一 \\
\hline \multirow{2}{*}{6.0} & $\mathrm{R}$ & - & - & - & $0.38 \pm 0.12$ & $0.53 \pm 0.04$ & $0.44 \pm 0.08$ & - & 一 \\
\hline & G & $0.58 \pm 0.12$ & $0.98 \pm 0.08$ & $1.54 \pm 0.12$ & $2.58 \pm 0.04$ & $3.36 \pm 0.12$ & $2.84 \pm 0.18$ & $1.92 \pm 0.24$ & $0.88 \pm 0.18$ \\
\hline \multirow{2}{*}{6.5} & $\mathrm{R}$ & $0.24 \pm 0.08$ & $0.42 \pm 0.04$ & $0.54 \pm 0.12$ & $1.26 \pm 0.08$ & $1.08 \pm 0.16$ & $0.49 \pm 0.10$ & $0.32 \pm 0.04$ & - \\
\hline & G & $0.36 \pm 0.06$ & $0.56 \pm 0.14$ & $2.62 \pm 0.28$ & $3.35 \pm 0.12$ & $4.66 \pm 0.28$ & $2.28 \pm 0.18$ & $1.88 \pm 0.26$ & $0.96 \pm 0.14$ \\
\hline \multirow{2}{*}{7.0} & $\mathrm{R}$ & - & $0.32 \pm 0.18$ & $1.41 \pm 0.22$ & $1.88 \pm 0.28$ & $1.35 \pm 0.12$ & $0.96 \pm 0.18$ & $0.48 \pm 0.18$ & - \\
\hline & G & $0.48 \pm 0.06$ & $0.88 \pm 0.14$ & $2.84 \pm 0.22$ & $4.62 \pm 0.40$ & $5.84 \pm 0.32$ & $3.56 \pm 0.24$ & $1.54 \pm 0.12$ & $0.98 \pm 0.12$ \\
\hline \multirow{2}{*}{7.5} & $\mathrm{R}$ & - & $0.24 \pm 0.06$ & $0.48 \pm 0.12$ & $0.96 \pm 0.12$ & $1.54 \pm 0.10$ & $0.78 \pm 0.36$ & $0.32 \pm 0.18$ & - \\
\hline & G & $0.88 \pm 0.26$ & $1.94 \pm 0.16$ & $2.86 \pm 0.28$ & $3.76 \pm 0.22$ & $4.88 \pm 0.28$ & $2.45 \pm 0.24$ & $1.76 \pm 0.18$ & $1.12 \pm 0.16$ \\
\hline \multirow{2}{*}{8.0} & $\mathrm{R}$ & - & - & $0.34 \pm 0.02$ & $0.56 \pm 0.08$ & $0.86 \pm 0.20$ & $0.46 \pm 0.12$ & - & - \\
\hline & G & - & $0.48 \pm 0.14$ & $0.84 \pm 0.18$ & $1.66 \pm 0.10$ & $2.56 \pm 0.16$ & $1.86 \pm 0.22$ & $0.92 \pm 0.02$ & $0.58 \pm 0.20$ \\
\hline
\end{tabular}

R: resting cells; G: growing cells; -: no hydrogen production.

TABLE 2: Effect of carbon sources on hydrogen production ( $\mathrm{mL} / 15 \mathrm{~mL}$ vessel) by $R$. palustris under anaerobic light.

\begin{tabular}{|c|c|c|c|c|c|c|c|c|c|}
\hline \multirow{2}{*}{ Carbon source } & & \multicolumn{6}{|c|}{ Incubation period (in hrs) } & \multirow[b]{2}{*}{166} & \multirow[b]{2}{*}{192} \\
\hline & & 24 & 48 & 72 & 96 & 120 & 144 & & \\
\hline \multirow{2}{*}{ Lactate } & $\mathrm{R}$ & $0.32 \pm 0.18$ & $0.66 \pm 0.04$ & $1.22 \pm 0.10$ & $1.65 \pm 0.10$ & $2.44 \pm 0.14$ & $1.72 \pm 0.28$ & $0.96 \pm 0.24$ & $0.48 \pm 0.24$ \\
\hline & G & $0.78 \pm 0.18$ & $1.14 \pm 0.22$ & $2.88 \pm 0.14$ & $4.10 \pm 0.36$ & $5.94 \pm 0.16$ & $4.52 \pm 0.28$ & $3.96 \pm 0.36$ & $2.88 \pm 0.14$ \\
\hline \multirow{2}{*}{ Malate } & $\mathrm{R}$ & $0.38 \pm 0.16$ & $0.76 \pm 0.26$ & $0.94 \pm 0.12$ & $1.78 \pm 0.22$ & $2.86 \pm 0.14$ & $1.96 \pm 0.08$ & $1.16 \pm 0.08$ & $0.78 \pm 0.16$ \\
\hline & G & $2.44 \pm 0.18$ & $3.74 \pm 0.14$ & $4.66 \pm 0.18$ & $5.14 \pm 0.46$ & $6.22 \pm 0.28$ & $5.42 \pm 0.16$ & $4.28 \pm 0.32$ & $3.66 \pm 0.24$ \\
\hline \multirow{2}{*}{ Succinate } & $\mathrm{R}$ & - & $0.22 \pm 0.04$ & $0.54 \pm 0.24$ & $0.88 \pm 0.18$ & $1.66 \pm 0.08$ & $1.10 \pm 0.10$ & $0.76 \pm 0.02$ & $0.38 \pm 0.10$ \\
\hline & G & $0.96 \pm 0.06$ & $1.84 \pm 0.18$ & $2.62 \pm 0.14$ & $3.88 \pm 0.36$ & $4.28 \pm 0.28$ & $3.44 \pm 0.18$ & $2.86 \pm 0.42$ & $1.76 \pm 0.32$ \\
\hline \multirow{2}{*}{ Fructose } & $\mathrm{R}$ & - & - & - & $0.34 \pm 0.08$ & $0.56 \pm 0.18$ & $0.22 \pm 0.06$ & - & - \\
\hline & G & - & - & $0.34 \pm 0.04$ & $0.66 \pm 0.12$ & $1.8 \pm 0.28$ & $0.82 \pm 0.04$ & $0.40 \pm 0.06$ & - \\
\hline \multirow{2}{*}{ Glucose } & $\mathrm{R}$ & - & - & - & - & $0.26 \pm 0.06$ & - & - & - \\
\hline & G & - & - & $0.28 \pm 0.12$ & $0.74 \pm 0.08$ & $1.25 \pm 0.04$ & $0.36 \pm 0.12$ & - & - \\
\hline \multirow{2}{*}{ Lactose } & $\mathrm{R}$ & - & - & $0.38 \pm 0.06$ & $0.64 \pm 0.06$ & $1.4 \pm 0.02$ & $0.88 \pm 0.06$ & $0.52 \pm 0.04$ & $0.26 \pm 0.08$ \\
\hline & G & $0.66 \pm 0.04$ & $1.22 \pm 0.08$ & $1.66 \pm 0.06$ & $2.78 \pm 0.20$ & $3.22 \pm 0.12$ & $2.56 \pm 0.16$ & $1.78 \pm 0.14$ & $0.94 \pm 0.08$ \\
\hline \multirow{2}{*}{ Maltose } & $\mathrm{R}$ & - & - & - & $0.28 \pm 0.18$ & $0.68 \pm 0.10$ & $0.38 \pm 0.20$ & - & - \\
\hline & G & $0.46 \pm 0.06$ & $0.84 \pm 0.10$ & $1.12 \pm 0.28$ & $1.88 \pm 0.32$ & $2.46 \pm 0.26$ & $1.36 \pm 0.32$ & $0.92 \pm 0.24$ & $0.62 \pm 0.12$ \\
\hline \multirow{2}{*}{ Sucrose } & $\mathrm{R}$ & - & - & - & $0.20 \pm 0.20$ & $0.72 \pm 0.16$ & $0.38 \pm 0.14$ & - & - \\
\hline & G & - & $0.40 \pm 0.20$ & $0.52 \pm 0.12$ & $0.88 \pm 0.12$ & $1.66 \pm 0.20$ & $1.22 \pm 0.22$ & $0.66 \pm 0.22$ & $0.28 \pm 0.04$ \\
\hline \multirow{2}{*}{ Mannitol } & $\mathrm{R}$ & - & - & $0.58 \pm 0.04$ & $0.98 \pm 0.12$ & $1.14 \pm 0.14$ & $0.76 \pm 0.12$ & $0.34 \pm 0.06$ & - \\
\hline & G & $0.56 \pm 0.18$ & $1.34 \pm 0.22$ & $2.12 \pm 0.20$ & $2.68 \pm 0.44$ & $3.86 \pm 0.28$ & $2.78 \pm 0.16$ & $1.86 \pm 0.08$ & $0.78 \pm 0.12$ \\
\hline \multirow{2}{*}{ Sorbitol } & $\mathrm{R}$ & - & - & - & $0.28 \pm 0.08$ & $0.44 \pm 0.12$ & - & - & - \\
\hline & G & - & $0.26 \pm 0.10$ & $0.52 \pm 0.12$ & $0.84 \pm 0.08$ & $1.54 \pm 0.06$ & $0.66 \pm 0.18$ & $0.44 \pm 0.04$ & - \\
\hline
\end{tabular}

R: resting cells; G: growing cells; - : no hydrogen production.

sources were tested, acetate was used as a source of carbon. Similar conditions were maintained devoid of light for investigations under anaerobic dark conditions.

2.2. Preparation of Resting Cell Suspensions. Cells of $R$. palustris were grown with acetate and ammonium chloride until mid-log phase and were harvested by centrifugation $(16,000 \times \mathrm{g}$ for 20 minutes). The pellet was washed twice and resuspended in basal salts medium. This suspension was then distributed into screw-cap test tubes $(10 \times 100 \mathrm{~mm})$ to fill them fully (anaerobic) or into $20 \mathrm{~mL}$ medium in a $100 \mathrm{~mL}$ conical flask (aerobic) and incubated under light $(2,400$ lux $)$ or dark conditions at $32^{\circ} \mathrm{C}$. Similar conditions were maintained devoid of light for investigations under anaerobic dark conditions.

The basic technique used in the hydrogen production was that of Vincenzini et al. [10]. Five mL of bacterial culture was harvested by centrifugation at $10,000 \times$ g for $10 \mathrm{~min}$, washed 
TABLE 3: Effect of nitrogen sources on hydrogen production $(\mathrm{mL} / 15 \mathrm{~mL}$ vessel) by $R$. palustris under anaerobic light.

\begin{tabular}{|c|c|c|c|c|c|c|c|c|c|}
\hline \multirow{2}{*}{ Nitrogen source } & & \multicolumn{6}{|c|}{ Incubation period (in hrs) } & \multirow[b]{2}{*}{166} & \multirow[b]{2}{*}{192} \\
\hline & & 24 & 48 & 72 & 96 & 120 & 144 & & \\
\hline \multirow{2}{*}{ Potassium nitrate } & $\mathrm{R}$ & - & - & $0.22 \pm 0.04$ & $0.42 \pm 0.08$ & $0.66 \pm 0.10$ & $0.36 \pm 0.08$ & & \\
\hline & G & - & - & $0.46 \pm 0.08$ & $0.88 \pm 0.04$ & $1.22 \pm 0.10$ & $0.62 \pm 0.18$ & $0.32 \pm 0.04$ & - \\
\hline \multirow{2}{*}{ Sodium nitrate } & $\mathrm{R}$ & - & - & $0.36 \pm 0.06$ & $0.52 \pm 0.08$ & $0.76 \pm 0.10$ & $0.48 \pm 0.12$ & - & - \\
\hline & G & $0.42 \pm 0.08$ & $0.54 \pm 0.14$ & $0.84 \pm 0.12$ & $1.26 \pm 0.14$ & $1.86 \pm 0.18$ & $1.44 \pm 0.28$ & $1.08 \pm 0.16$ & $0.66 \pm 0.06$ \\
\hline \multirow{2}{*}{ Ammonium chloride } & $\mathrm{R}$ & - & - & - & - & - & - & - & - \\
\hline & G & - & - & - & - & - & - & - & - \\
\hline \multirow{2}{*}{ Urea } & $\mathrm{R}$ & - & - & - & - & $0.34 \pm 0.04$ & - & - & - \\
\hline & G & - & - & $0.38 \pm 0.08$ & $0.66 \pm 0.08$ & $0.88 \pm 0.12$ & $0.52 \pm 0.16$ & $0.38 \pm 0.04$ & - \\
\hline \multirow{2}{*}{ Thiourea } & $\mathrm{R}$ & - & - & - & - & - & & & - \\
\hline & G & - & - & - & $0.38 \pm 0.18$ & $0.54 \pm 0.28$ & $0.34 \pm 0.02$ & $0.22 \pm 0.06$ & - \\
\hline \multirow{2}{*}{ Glycine } & $\mathrm{R}$ & & - & - & $0.28 \pm 0.08$ & $0.48 \pm 0.10$ & $0.36 \pm 0.06$ & - & - \\
\hline & G & - & - & $0.54 \pm 0.20$ & $0.96 \pm 0.12$ & $1.3 \pm 0.18$ & $0.74 \pm 0.14$ & $0.46 \pm 0.10$ & - \\
\hline \multirow{2}{*}{ L-asparagine } & $\mathrm{R}$ & - & - & $0.24 \pm 0.16$ & $0.44 \pm 0.12$ & $0.66 \pm 0.08$ & $0.36 \pm 0.06$ & - & - \\
\hline & G & - & $0.46 \pm 0.02$ & $0.84 \pm 0.12$ & $1.26 \pm 0.06$ & $1.74 \pm 0.18$ & $1.46 \pm 0.12$ & $1.16 \pm 0.06$ & $0.68 \pm 0.06$ \\
\hline \multirow{2}{*}{ L-aspartic acid } & $\mathrm{R}$ & - & - & $0.36 \pm 0.18$ & $0.68 \pm 0.18$ & $0.96 \pm 0.08$ & $0.58 \pm 0.20$ & $0.26 \pm 0.08$ & - \\
\hline & G & $0.52 \pm 0.06$ & $0.76 \pm 0.08$ & $1.34 \pm 0.08$ & $1.84 \pm 0.08$ & $2.2 \pm 0.08$ & $1.54 \pm 0.08$ & $1.08 \pm 0.18$ & $0.66 \pm 0.12$ \\
\hline \multirow{2}{*}{ L-glutamic acid } & $\mathrm{R}$ & - & - & $0.66 \pm 0.04$ & $0.88 \pm 0.08$ & $1.22 \pm 0.10$ & $0.98 \pm 0.12$ & $0.72 \pm 0.04$ & - \\
\hline & G & $0.48 \pm 0.10$ & $0.96 \pm 0.16$ & $1.54 \pm 0.12$ & $1.86 \pm 0.08$ & $2.56 \pm 0.06$ & $2.22 \pm 0.08$ & $1.76 \pm 0.08$ & $0.84 \pm 0.08$ \\
\hline \multirow{2}{*}{ L-glutamine } & $\mathrm{R}$ & - & - & - & $0.48 \pm 0.12$ & $0.78 \pm 0.04$ & $0.56 \pm 0.06$ & $0.34 \pm 0.10$ & 一 \\
\hline & G & - & $0.42 \pm 0.08$ & $0.76 \pm 0.14$ & $1.16 \pm 0.24$ & $1.58 \pm 0.16$ & $1.24 \pm 0.28$ & $0.88 \pm 0.14$ & $0.54 \pm 0.02$ \\
\hline \multirow{2}{*}{ L-tyrosine } & $\mathrm{R}$ & - & - & $0.32 \pm 0.02$ & $0.46 \pm 0.22$ & $0.84 \pm 0.28$ & $0.58 \pm 0.06$ & $0.28 \pm 0.14$ & - \\
\hline & G & $0.38 \pm 0.04$ & $0.78 \pm 0.06$ & $1.18 \pm 0.14$ & $1.56 \pm 0.14$ & $2.30 \pm 0.08$ & $1.78 \pm 0.12$ & $1.34 \pm 0.16$ & $0.58 \pm 0.04$ \\
\hline \multirow{2}{*}{ L-cystine } & $\mathrm{R}$ & - & - & $0.32 \pm 0.04$ & $0.54 \pm 0.08$ & $1.10 \pm 0.08$ & $0.68 \pm 0.12$ & $0.38 \pm 0.14$ & - \\
\hline & G & $0.30 \pm 0.04$ & $0.76 \pm 0.10$ & $1.34 \pm 0.12$ & $1.64 \pm 0.14$ & $1.94 \pm 0.28$ & $1.48 \pm 0.24$ & $1.10 \pm 0.22$ & $0.54 \pm 0.16$ \\
\hline \multirow{2}{*}{ L-methionine } & $\mathrm{R}$ & - & - & - & $0.34 \pm 0.06$ & $0.64 \pm 0.04$ & $0.40 \pm 0.08$ & - & - \\
\hline & G & - & - & $0.46 \pm 0.04$ & $0.84 \pm 0.32$ & $1.24 \pm 0.26$ & $0.96 \pm 0.10$ & $0.56 \pm 0.12$ & 一 \\
\hline \multirow{2}{*}{ Nitrogen gas } & $\mathrm{R}$ & - & - & - & - & $0.28 \pm 0.02$ & - & - & - \\
\hline & G & - & - & $0.28 \pm 0.10$ & $0.66 \pm 0.18$ & $1.12 \pm 0.32$ & $0.42 \pm 0.16$ & - & 一 \\
\hline
\end{tabular}

R: resting cells; G: growing cells; 一: no hydrogen production.

thrice with $0.3 \%$ saline, and the cells were suspended in the basal medium devoid of electron donor and nitrogen source. Depending on the experimental conditions, different electron donors and nitrogen sources were added at required concentrations. To test the hydrogen production activity, the washed cell suspension was inoculated into $8 \mathrm{~mL}$ of the medium in $15 \mathrm{~mL}$ capacity rimless test tubes sealed with subaseals, and anaerobic conditions were created by evacuating and flushing with nitrogen (100\%). Cumulative hydrogen production was recorded at various time intervals. Hydrogen produced was measured by injecting $0.5 \mathrm{~mL}$ of the gas phase from the reaction vessels with an airtight syringe into a gas chromatograph (Mak Analytica make) fitted with a molecular sieve $5 \mathrm{~A}$ column $\left(2 \mathrm{~m} \times 1 / 8^{\prime \prime}\right.$ ODSS $)$ to a thermal conductivity detector (TCD). Gas analysis was done with oven temperature at $60^{\circ} \mathrm{C}$ with argon as carrier gas (flow rate $30 \mathrm{~mL} / \mathrm{min}$ ), $120 \mathrm{~mA}$ detector current. Integrator and recorder were used at highest sensitivity. Before withdrawing each sample, $0.5 \mathrm{~mL}$ of nitrogen was injected in the vessel to maintain positive pressure. The amount of hydrogen liberated by the photosynthetic bacterium was calculated from the peak height of the recorder with reference to calibration curve prepared using ultrapure hydrogen.

\section{Results and Discussion}

Perusal of Table 1 reveals that $R$. palustris could produce hydrogen over a narrow $\mathrm{pH}$ range of 5.0 to 8.0. Growth of the organism started from $\mathrm{pH} 4.00$ but hydrogen production could not be recorded till $\mathrm{pH}$ 5.0, hence data was not shown. Rps. palustris opted for $\mathrm{pH} 7.0$ for maximum production of hydrogen by growing cells and resting cells. Incubation period of 96-120 hrs was optimum for production of hydrogen. There were variations in the initial $\mathrm{pH}$ and final $\mathrm{pH}$ but the variations were minute and not greater than $0.28( \pm)$. Hence, they were not presented in the table. From Table 2, it is clear that the bacteria under investigation showed preference towards carbon source present in the medium. Rps. palustris preferred malate for maximum production of hydrogen at $120 \mathrm{hrs}$ incubation. Lactate and Succinate also promoted more amounts of hydrogen production under anaerobic light. Glucose promoted lowest amounts of hydrogen production. 
TABLE 4: Effect of carbon sources on hydrogen production ( $\mathrm{mL} / 15 \mathrm{~mL}$ vessel) by $R$. palustris under anaerobic dark.

\begin{tabular}{|c|c|c|c|c|c|c|c|c|c|}
\hline \multirow{2}{*}{\multicolumn{2}{|c|}{ Carbon source }} & \multicolumn{6}{|c|}{ Incubation period (in hrs) } & \multirow[b]{2}{*}{166} & \multirow[b]{2}{*}{192} \\
\hline & & 24 & 48 & 72 & 96 & 120 & 144 & & \\
\hline \multirow{2}{*}{ Lactate } & $\mathrm{R}$ & - & - & $0.24 \pm 0.02$ & $0.48 \pm 0.10$ & $0.66 \pm 0.14$ & $0.38 \pm 0.18$ & - & - \\
\hline & G & - & - & $0.38 \pm 0.16$ & $0.68 \pm 0.18$ & $1.24 \pm 0.12$ & $0.84 \pm 0.28$ & $0.44 \pm 0.12$ & $0.32 \pm 0.18$ \\
\hline \multirow{2}{*}{ Malate } & $\mathrm{R}$ & - & - & $0.42 \pm 0.06$ & $0.68 \pm 0.32$ & $0.98 \pm 0.02$ & $0.36 \pm 0.16$ & - & - \\
\hline & G & $0.36 \pm 0.14$ & $0.58 \pm 0.14$ & $0.98 \pm 0.14$ & $1.26 \pm 0.24$ & $1.68 \pm 0.20$ & $1.34 \pm 0.32$ & $0.84 \pm 0.14$ & $0.40 \pm 0.18$ \\
\hline \multirow{2}{*}{ Succinate } & $\mathrm{R}$ & - & - & - & $0.26 \pm 0.18$ & $0.54 \pm 0.12$ & $0.36 \pm 0.02$ & - & - \\
\hline & G & - & $0.26 \pm 0.06$ & $0.52 \pm 0.12$ & $0.78 \pm 0.04$ & $1.10 \pm 0.28$ & $0.82 \pm 0.18$ & $0.68 \pm 0.06$ & $0.38 \pm 0.20$ \\
\hline \multirow{2}{*}{ Fructose } & $\mathrm{R}$ & - & - & - & - & $0.22 \pm 0.08$ & - & - & - \\
\hline & G & - & - & $0.46 \pm 0.14$ & $0.56 \pm 0.20$ & $0.78 \pm 0.28$ & $0.38 \pm 0.12$ & $0.28 \pm 0.08$ & - \\
\hline \multirow{2}{*}{ Glucose } & $\mathrm{R}$ & - & - & - & - & $0.28 \pm 0.06$ & - & - & - \\
\hline & G & - & - & - & $0.38 \pm 0.10$ & $0.56 \pm 0.14$ & $0.26 \pm 0.04$ & - & - \\
\hline \multirow{2}{*}{ Lactose } & $\mathrm{R}$ & - & - & - & $0.48 \pm 0.08$ & $0.74 \pm 0.12$ & $0.36 \pm 0.03$ & - & - \\
\hline & G & - & $0.34 \pm 0.16$ & $0.68 \pm 0.06$ & $1.04 \pm 0.28$ & $1.48 \pm 0.16$ & $0.92 \pm 0.14$ & $0.76 \pm 0.28$ & $0.54 \pm 0.18$ \\
\hline \multirow{2}{*}{ Maltose } & $\mathrm{R}$ & - & - & - & - & $0.38 \pm 0.10$ & - & - & - \\
\hline & G & - & - & $0.22 \pm 0.14$ & $0.44 \pm 0.32$ & $0.94 \pm 0.14$ & $0.56 \pm 0.10$ & $0.32 \pm 0.18$ & - \\
\hline \multirow{2}{*}{ Sucrose } & $\mathrm{R}$ & - & - & - & $0.24 \pm 0.12$ & $0.48 \pm 0.06$ & $0.28 \pm 0.18$ & - & - \\
\hline & G & - & - & $0.34 \pm 0.02$ & $0.66 \pm 0.18$ & $1.18 \pm 0.24$ & $0.78 \pm 0.32$ & $0.46 \pm 0.12$ & $0.26 \pm 0.18$ \\
\hline \multirow{2}{*}{ Mannitol } & $\mathrm{R}$ & - & - & - & - & $0.40 \pm 0.04$ & - & - & - \\
\hline & G & - & - & - & $0.42 \pm 0.06$ & $0.64 \pm 0.16$ & $0.38 \pm 0.08$ & - & - \\
\hline \multirow{2}{*}{ Sorbitol } & $\mathrm{R}$ & - & - & - & $0.36 \pm 0.22$ & $0.68 \pm 0.20$ & $0.42 \pm 0.12$ & $0.26 \pm 0.06$ & - \\
\hline & G & - & - & $0.32 \pm 0.02$ & $0.54 \pm 0.08$ & $0.86 \pm 0.14$ & $0.40 \pm 0.08$ & $0.24 \pm 0.04$ & - \\
\hline
\end{tabular}

R: resting cells; G: growing cells; - : no hydrogen production.

L-glutamic acid followed by L-tyrosine and L-aspartic acid were good promoters of hydrogen in Rps. palustris in anaerobic light (Table 3). Ammonium chloride failed to produce hydrogen. Glycine and potassium nitrate were of equal nutritive value for the production of hydrogen. Growing cells produced more amount of hydrogen than resting cells. Thiourea promoted less amounts of hydrogen and was a poor nitrogen source for hydrogen production by Rps. palustris. Hydrogen production started from $24 \mathrm{hrs}$ and considerable amount still could be recorded by the end of $192 \mathrm{hrs}$ in glutamic-acid-containing medium. Perusal of Table 4 shows that malate and Lactose were preferred over other carbon sources under anaerobic dark conditions. Maltose and sorbitol were of same nutritive value for the production of hydrogen. In glucose, fructose, and maltose medium production of hydrogen was observed only at $120 \mathrm{hrs}$ in growing cells. Lowest amounts of hydrogen were recorded in glucose medium similar to that in anaerobic light conditions. Hydrogen production was less in anaerobic dark conditions when compared to anaerobic light conditions. Growing cells could produce more amount of hydrogen in malate medium over extended periods. The hydrogen production started early in growing cells than in resting cells in most of the carbon sources tried.

Ammonium chloride failed to produce hydrogen production in Rps. palustris under anaerobic dark (Table 5). Inhibition of hydrogen in presence of ammonium ions has also been reported by Salerno et al. [11]. Thiourea, urea, and nitrogen gas were responsible for inhibition of hydrogen production in resting cells. In all other nitrogen sources tried, growing cells produced more hydrogen than resting cells. Resting cells in general showed a lag in hydrogen production than growing cells of Rps. palustris. Highest production of hydrogen was recorded in growing cells of L-glutamic acid and tyrosine media.

Carbon sources are known to influence hydrogen production through nitrogenase enzyme by causing variation in electron donation capabilities of the cofactor compounds to nitrogenase [12]. Hence, differences in hydrogen production rates with different carbon sources were observed. In our study, organic nitrogen sources produced more amounts of hydrogen compared to inorganic nitrogen sources. Organic nitrogen sources are directly incorporated into proteins or transformed into other cellular nitrogenous constituents [13]. In contrast, cell spends more time in synthesizing amino acids for protein synthesis from inorganic nitrogen sources. This might be the reason for the variation observed in hydrogen production rates. Further studies are required to understand the molecular mechanism behind these observed variations in production of hydrogen with different carbon and nitrogen sources.

\section{Conclusions}

The present study show the ability of this phototrophic bacterium to produce hydrogen in different cultural conditions which can be exploited. Anaerobic dark conditions required to be explored more as it is more economically attractive. Investigations are on to find out more suitable and cheaper 
TABLE 5: Effect of nitrogen sources on hydrogen production $(\mathrm{mL} / 15 \mathrm{~mL}$ vessel) by $R$. palustris under anaerobic dark.

\begin{tabular}{|c|c|c|c|c|c|c|c|c|c|}
\hline \multirow{2}{*}{ Nitrogen source } & & \multicolumn{6}{|c|}{ Incubation period (in hrs) } & \multirow[b]{2}{*}{166} & \multirow[b]{2}{*}{192} \\
\hline & & 24 & 48 & 72 & 96 & 120 & 144 & & \\
\hline \multirow{2}{*}{ Potassium nitrate } & $\mathrm{R}$ & - & - & - & - & $0.22 \pm 0.06$ & - & - & - \\
\hline & G & - & - & $0.38 \pm 0.04$ & $0.68 \pm 0.06$ & $0.98 \pm 0.08$ & $0.74 \pm 0.18$ & $0.30 \pm 0.18$ & - \\
\hline \multirow{2}{*}{ Sodium nitrate } & $\mathrm{R}$ & - & - & - & - & $0.23 \pm 0.10$ & - & - & - \\
\hline & G & - & - & - & $0.30 \pm 0.04$ & $0.46 \pm 0.18$ & $0.22 \pm 0.08$ & - & - \\
\hline \multirow{2}{*}{ Ammonium chloride } & $\mathrm{R}$ & - & - & - & - & - & - & - & - \\
\hline & G & - & - & - & - & - & - & - & - \\
\hline \multirow{2}{*}{ Urea } & $\mathrm{R}$ & - & - & - & - & - & - & - & - \\
\hline & G & - & - & - & $0.24 \pm 0.08$ & $0.38 \pm 0.02$ & - & - & - \\
\hline \multirow{2}{*}{ Thiourea } & $\mathrm{R}$ & - & - & - & - & - & - & - & - \\
\hline & G & - & - & - & - & $0.24 \pm 0.08$ & - & - & - \\
\hline \multirow{2}{*}{ Glycine } & $\mathrm{R}$ & - & - & - & - & $0.28 \pm 0.06$ & - & - & - \\
\hline & G & - & - & - & $0.38 \pm 0.12$ & $0.74 \pm 0.16$ & $0.56 \pm 0.04$ & $0.22 \pm 0.18$ & - \\
\hline \multirow{2}{*}{ L-asparagine } & $\mathrm{R}$ & - & - & - & - & $0.36 \pm 0.04$ & - & - & - \\
\hline & G & - & $0.38 \pm 0.06$ & $0.76 \pm 0.12$ & $0.98 \pm 0.10$ & $1.04 \pm 0.08$ & $0.74 \pm 0.18$ & $0.42 \pm 0.06$ & - \\
\hline \multirow{2}{*}{ L-glutamic acid } & $\mathrm{R}$ & - & - & - & - & $0.36 \pm 0.08$ & - & - & - \\
\hline & G & - & - & $0.64 \pm 0.18$ & $0.94 \pm 0.08$ & $1.6 \pm 0.28$ & $0.72 \pm 0.14$ & $0.54 \pm 0.10$ & - \\
\hline \multirow{2}{*}{ L-glutamine } & $\mathrm{R}$ & - & - & - & - & $0.52 \pm 0.08$ & $0.30 \pm 0.02$ & - & - \\
\hline & G & $0.48 \pm 0.14$ & $0.72 \pm 0.08$ & $0.98 \pm 0.16$ & $1.22 \pm 0.08$ & $1.16 \pm 0.30$ & $1.14 \pm 0.06$ & $0.86 \pm 0.26$ & $0.44 \pm 0.12$ \\
\hline \multirow{2}{*}{ L-tyrosine } & $\mathrm{R}$ & - & - & - & $0.32 \pm 0.02$ & $0.48 \pm 0.10$ & $0.24 \pm 0.04$ & - & - \\
\hline & G & - & - & - & $0.28 \pm 0.06$ & $0.46 \pm 0.08$ & $0.34 \pm 0.04$ & - & - \\
\hline \multirow{2}{*}{ L-cystine } & $\mathrm{R}$ & - & - & - & - & $0.28 \pm 0.08$ & - & - & - \\
\hline & G & - & - & - & $0.38 \pm 0.14$ & $0.56 \pm 0.04$ & $0.34 \pm 0.02$ & - & - \\
\hline \multirow{2}{*}{ L-methionine } & $\mathrm{R}$ & - & - & - & - & $0.38 \pm 0.18$ & - & - & - \\
\hline & G & - & $0.20 \pm 0.02$ & $0.44 \pm 0.08$ & $0.72 \pm 0.12$ & $0.86 \pm 0.14$ & $0.56 \pm 0.22$ & $0.38 \pm 0.06$ & - \\
\hline \multirow{2}{*}{ Nitrogen gas } & $\mathrm{R}$ & - & - & - & - & - & - & - & - \\
\hline & G & - & - & - & - & $0.42 \pm 0.12$ & - & - & - \\
\hline \multirow{2}{*}{ L-aspartic acid } & $\mathrm{R}$ & - & - & - & $0.38 \pm 0.12$ & $0.48 \pm 0.02$ & $0.58 \pm 0.06$ & $0.26 \pm 0.08$ & - \\
\hline & G & - & $0.46 \pm 0.04$ & $0.74 \pm 0.08$ & $1.04 \pm 0.10$ & $0.96 \pm 0.12$ & $0.76 \pm 0.22$ & $0.54 \pm 0.26$ & $0.34 \pm 0.08$ \\
\hline
\end{tabular}

R: resting cells; G: growing cells; 一: no hydrogen production.

carbon and nitrogen sources for hydrogen production. Further, the organism should be investigated for simultaneous production of PHB (polyhydroxy butyrate) and ALA (amino levulinic acid) along with the production of hydrogen.

\section{References}

[1] S. P. Singh, S. C. Srivastava, V. K. Singh, D. L. Block, and T. N. Veziroglu, "Photoproduction of hydrogen by Rhodopseudomonas at the expense of cellulose: development of two stage bioreactor," in Proceedings of the 10th World Hydrogen Energy Conference, vol. 2, pp. 959-963, Cocoa beach, Fla, USA, 1994.

[2] X. Wang, B. Jin, and D. Mulcahy, "Impact of carbon and nitrogen sources on hydrogen production by a newly isolated Clostridium butyricum W5," International Journal of Hydrogen Energy, vol. 33, no. 19, pp. 4998-5005, 2008.

[3] Ch. Sasikala and Ch. V. Ramana, "Biotechnological potentials of anoxygenic phototrophic bacteria. I. Production of singlecell protein, vitamins, ubiquinones, hormones, and enzymes and use in waste treatment," Advances in Applied Microbiology, vol. 41, pp. 173-226, 1995.
[4] Y. Tao, Y. Chen, Y. Wu, Y. He, and Z. Zhou, "High hydrogen yield from a two-step process of dark- and photo-fermentation of sucrose," International Journal of Hydrogen Energy, vol. 32, no. 2, pp. 200-206, 2007.

[5] T. Katsuda, N. Fujii, N. Takata, H. Ooshima, and S. Katoh, "Light attenuation in suspension of the purple bacterium Rhodobacter capsulatus and the green alga Chlamydomonas reinhardtii," Journal of Chemical Engineering of Japan, vol. 35, no. 5, pp. 428-435, 2002.

[6] G. Najafpour, H. Younesi, and A. R. Mohamed, "Effect of organic substrate on hydrogen production from synthesis gas using Rhodospirillum rubrum, in batch culture," Biochemical Engineering Journal, vol. 21, no. 2, pp. 123-130, 2004.

[7] M. R. Melnicki, L. Bianchi, R. De Philippis, and A. Melis, "Hydrogen production during stationary phase in purple photosynthetic bacteria," International Journal of Hydrogen Energy, vol. 33, no. 22, pp. 6525-6534, 2008.

[8] O. Mizuno, R. Dinsdale, F. R. Hawkes, D. L. Hawkes, and T. Noike, "Enhancement of hydrogen production from glucose by nitrogen gas sparging," Bioresource Technology, vol. 73, no. 1, pp. 59-65, 2000.

[9] J. T. Staley, M. P. Byrant, N. Pfennig, and J. C. Holt, Eds., "Enrichment and isolation of purple non sulphur photosyn- 
thetic bacteria," 1994, Bergey's Manual of Systematic Bacteriology.

[10] M. Vincenzini, R. Materassi, M. R. Tredici, and G. Florenzano, "Hydrogen production by immobilized cells-I. light dependent dissimilation of organic substances by Rhodopseudomonas palustris," International Journal of Hydrogen Energy, vol. 7, no. 3, pp. 231-236, 1982.

[11] M. B. Salerno, W. Park, Y. Zuo, and B. E. Logan, "Inhibition of biohydrogen production by ammonia," Water Research, vol. 40, no. 6, pp. 1167-1172, 2006.

[12] D. Madamwar, N. Garg, and V. Shah, "Cyanobacterial hydrogen production," World Journal of Microbiology and Biotechnology, vol. 16, no. 8-9, pp. 757-767, 2000.

[13] M. Ferchichi, E. Crabbe, W. Hintz, G. H. Gil, and A. Almadidy, "Influence of culture parameters on biological hydrogen production by Clostridium saccharoperbutylacetonicum ATCC 27021," World Journal of Microbiology and Biotechnology, vol. 21, no. 6-7, pp. 855-862, 2005. 

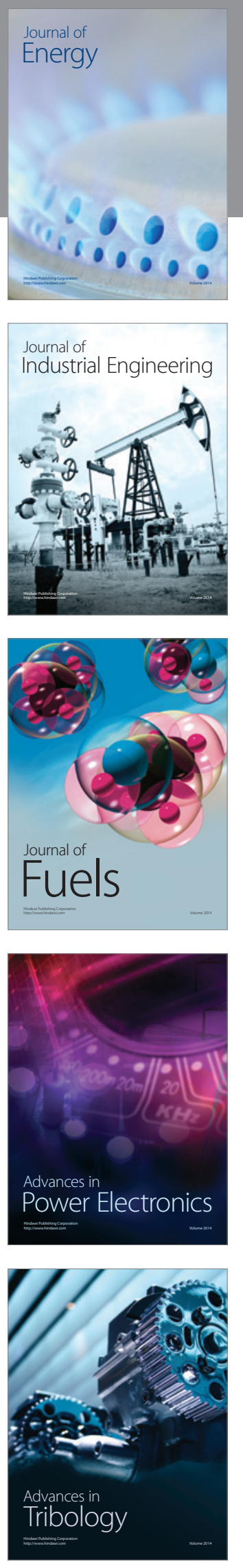
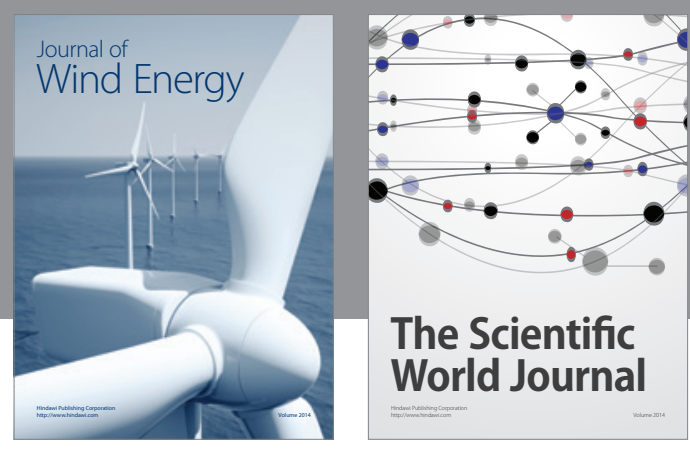

The Scientific World Journal

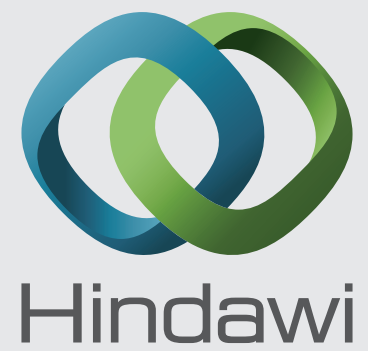

Submit your manuscripts at http://www.hindawi.com
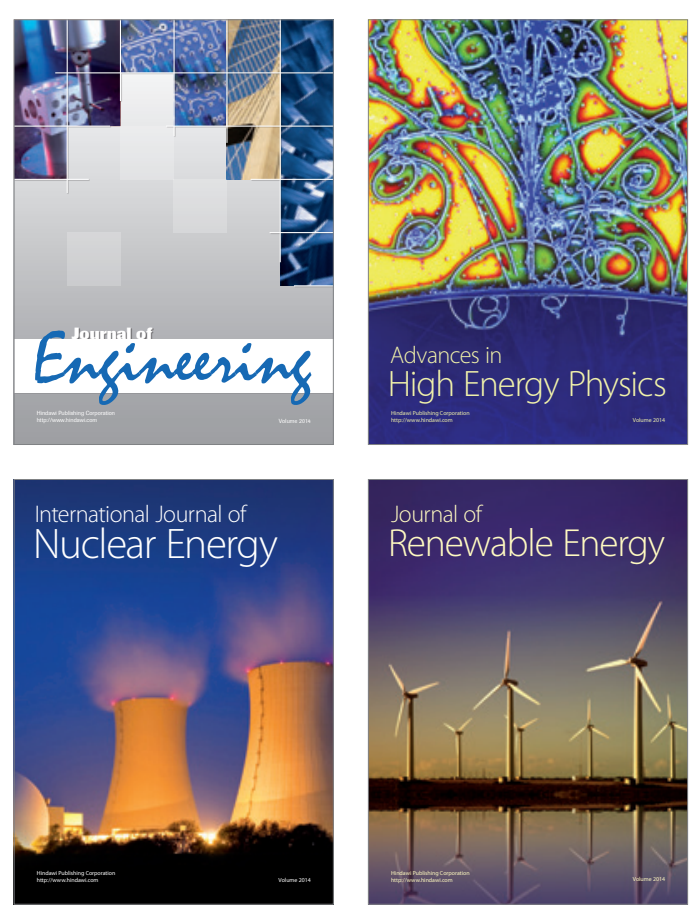

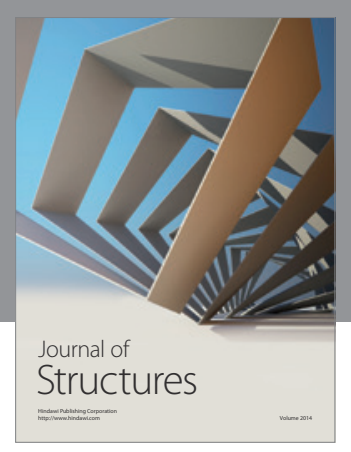

Rotating
Mechinery
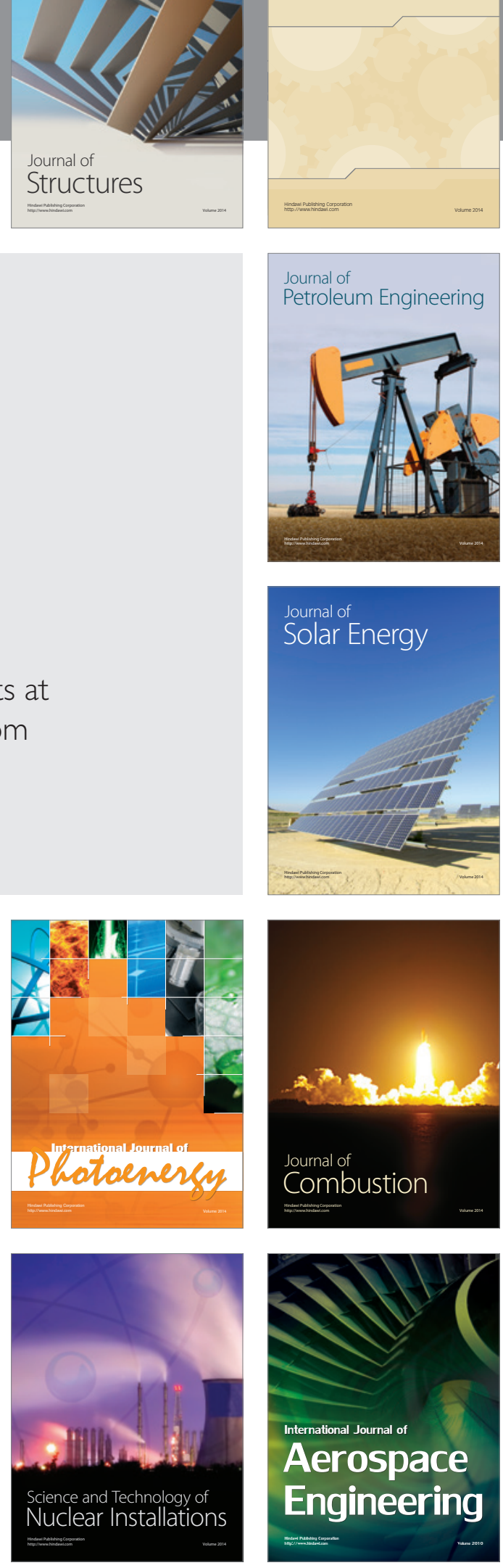